\title{
Multicriteria Decision-making Method for Scheduling Problem Based on Smart Batches and their Quality Prediction Capability
}

\author{
Emmanuel ZIMMERMANN ${ }^{1,2}$, Tsegay Tesfay MEZGEBE ${ }^{1,3}$, Hind BRIL EL HAOUZI $^{1}$, \\ Philippe THOMAS ${ }^{1 *}$, Rémi PANNEQUIN ${ }^{1}$, Mélanie NOYEL ${ }^{2}$

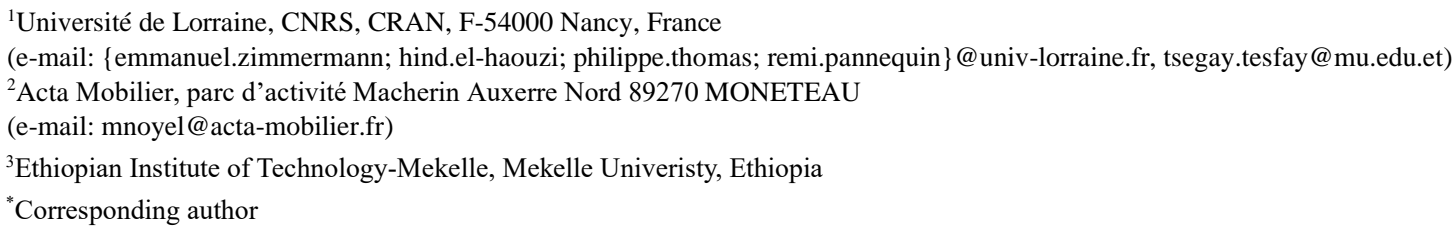

\begin{abstract}
The scheduling problem in manufacturing companies with high rework rates remains a complex research area to date. This paper presents a new approach for manufacturing scheduling that combines a predictive schedule with a proactive multicriteria decision-making method based on smart batches and their quality prediction capability. Each batch embeds an algorithm that allows it to predict its quality out of the next workstation. As soon as a batch determines that its process is too hazardous, a collaborative rescheduling decision, using the analytic hierarchy process (AHP), is initiated with its peer. This article details the proposed approach along with the AHP structure and presents the considered decision problem. A simulation model inspired by a lacquering-robot case study is described to validate this proposition. Then, the results of different scenarios are presented and discussed, highlighting the impact of social myopia on smart batches.
\end{abstract}

Keywords Proactive decision-making, Analytic hierarchy process (AHP), Multicriteria decision method, Quality prediction, Smart products, Rework.

\section{Introduction}

Scheduling problems have been addressed by some of the most important studies in manufacturing control management. Since 1960, many exact and heuristic approaches have been proposed in the literature to achieve the best outcomes, such as maximum profit or lowest cost (Hanssmann and Hess, 1960; Held and Karp, 1962). Classical techniques that determine globally optimized schedules are not very useful when facing disturbances, which is notably the case for companies suffering from high rework ${ }^{1}$ rates. This can be explained by the fact that as soon as a need for rework appears, the global schedule becomes obsolete because of the lack of agility of centralized/predictive solutions. To address this issue, two main paths could be explored: 1) robust scheduling methods and 2) distributed production scheduling methods. Motivated by the framework of Industry 4.0, the current focus is mainly on the second path. In the existing requirement for dynamic decision, the distribution of decisional ability to enable the system's self-adaptation to changing situations is seen as a great improvement vector for real-time problem-solving and adaptability enhancement (Mezgebe et al., 2019). However, such systems suffer from the phenomenon of social myopia, where decisional entities in distributed reactive decision-making cannot balance their local objectives with the system's

\footnotetext{
${ }^{1}$ See the works of Jamal et al. (2004) and Wee and Widyadana (2013) to define and clarify the meaning of rework.
} 
global objective (Rey et al., 2014). Based on the need for distributed decision processes and their inherent risk of social myopia, we identified a holonic architecture-based elaboration of the hybrid control system as the most promising approach owing to its ability to couple predictive and reactive approaches to achieve global optimization while limiting myopia (Cardin et al., 2017; Zimmermann et al., 2017).

In this paper, the scheduling problem of a lacquering-robot-based work center in a wood processing company, Acta Mobilier ${ }^{2}$, is considered. To be profitable in terms of both material consumption and the use of available time, the production consists of large batches. On the shop floor level, after lacquering, batches are directed to an oven for $7 \mathrm{~h}$ of drying. However, any non-quality work done during lacquering is only visible after the product is dried. Hence, if a poor-quality batch appears, it is only detected when leaving the oven $7 \mathrm{~h}$ after completion of the lacquering. Consequently, because it is very likely that the entire batch must be reworked, its process time is doubled and lengthened by another lacquering and drying process. Therefore, in previous studies, a prediction model of non-quality production risk was developed based on the use of a neural network (NN) (Noyel et al., 2016). Starting from this NN and its subsequent improvements (Thomas et al., 2018), we propose to use it as a means of triggering and reordering as well as for criterion selection involved in the analytic hierarchy process (AHP) decision-making process.

Further, a hybrid control system is proposed in this paper, coupling predictive scheduling and a proactive multicriteria decision method focused, in particular, on non-quality production risk. This is an extension of the work of Zimmermann et al. (2018) with significant improvements in problem modeling and including the presentation of a statistical study evaluating social myopia (number of active/smart batches in the decision-making process). This evaluation approach can be used to identify the optimal number of smart batches that provides a good trade-off between algorithm performance and results quality.

The rest of the paper is organized as follows: Section 2 presents research related to the contribution of hybrid manufacturing control systems to industrial real-time scheduling issues. Section 3 explains the proposed hybrid manufacturing control system coupling a predictive schedule with a proactive decisional process-based smart batch using the AHP method. Section 4 presents an industrial application by considering the proposed approach for the case study of a lacquering robot in the Acta Mobilier company. A simulation model is presented to validate the possible advantages of the proposed method. Experiments comparing the AHP-based decision-making process with actual decisional rule practices in the company are described. Finally, the impact of myopia on smart batch analysis is evaluated. The last section presents the conclusions and future prospects.

\footnotetext{
${ }^{2}$ Acta Mobilier is a French company in the wood industry. 


\section{Related work}

Today's globally volatile market is compelling manufacturing industries to adapt and react to different factors, such as requirements for highly flexible and value-added activities, highquality products, or shorter product life cycles. These new stakes have made centralized predictive schedulers obsolete, aggravating the flow disturbances due to non-quality in production processes. To meet these new requirements of high quality and flexibility, companies can either invest in state-of-the-art technologies and production tools or adapt their control architectures. Concerning the first option, we can consider the NN-based setup to limit non-quality as proposed by Noyel et al. (2016). The model depends on a multitude of both internal and external factors, such as actual workstation setups, production range of the next product to be processed, and environmental factors such as air humidity or temperature. However, the model proved to be insufficient because the considered system was working at technological limits. The second option, which could be combined with the first one, would be to try to predict the impact of non-quality products on production processes. For example, considering the same case study, Noyel et al. (2016) proposed a model based on a NN with the capability to predict the risk of non-quality for a particular product at a particular time. Using this capability, dynamical rescheduling can be performed to prevent non-quality, independently of technological limits.

This second option is supported by recent developments concerning hybrid control architectures. Most recent studies (such as Pach et al., 2014; Barbosa et al., 2015; Indriago et al., 2016; Quintanilla et al., 2016; Jimenez J. F. et al., 2017; Zimmermann et al., 2017; Valckenaers P., 2018; Mezgebe et al, 2019) have already proven the adequacy of such architectures to address resilience and adaptability issues for production flows. In their work, Zimmermann et al. (2017) proposed a hybrid architecture based on the product driven system concept, where an instance of the quality prediction NN model would be embedded on each product or batch, assuming that an optimized schedule was provided to workstations. This approach combines both global and local scheduling, the global one being established with no consideration for any rework problem and local scheduling being established for every work center, which follows its own optimization and is able to recalculate itself depending on the situation. Notably, as variant external factors such as atmospheric pressure or air humidity had an important impact on production in the studied case, products were able to decide to alter their schedule. Another characteristic of the architecture proposed by Zimmermann et al. (2017) is that products in the workstation queue must normally be processed following the optimal global schedule. Still, to prevent non-quality, local rescheduling might be performed under peculiar conditions by the products, and thanks to the risk prediction process.

To model and implement this hybrid architecture, many collective decision-making approaches executed in multi-agent systems have been introduced. Some of them are inspired by social attitudes such as consensus (Mezgebe et al., 2019) and majority voting (Pitt et al., 2006), or from the domain of game theory (Parsons and Wooldridge, 2002). Others seek to imitate biological behaviors such as swarm robotics (Schmickl et al., 2009), bee swarms (Teodorovic, 2003), and ant colonies (Xiang and Lee, 2008). In addition, the use of the AHP offers advantages to decision-makers for prioritization. The AHP relies on the establishment of a hierarchical structure to deal with complex decision-making and is able to consider all the criteria appearing in the decision-making process. The method is typically structured with 
several levels; some are mandatory: the global objective (level 0) and alternatives (last level). Between these two ends, there is a variable number of potential levels (named sub-criteria). The AHP method uses pairwise comparisons of same-level elements whose relative importance is evaluated at a higher level; a value scale is used to express the degree of importance of one element compared to another.

The adequacy of the AHP method for solving complex business decisions has already been well established in previous studies (Cheng et al., 2002; Vaidya and Kumar, 2006; Saaty, 2008; Liu and Hai, 2005; Bian et al., 2017; Moslem et al., 2019). Büyüközkan et al. (2021) stated that the AHP (Saaty, 1980) is the most utilized multicriteria decision-making method. Likewise, the AHP has been used for scheduling problems in the last two decades. Notably, Momoh and Zhu (2003) proposed an application of the AHP and analytic network process to prioritize, schedule, and optimize power unit price allocations. Later, Chan et al. (2005) coupled an AHP to a genetic algorithm to solve the production-distribution problem, while Azadeh et al. (2008) combined the AHP to data envelopment analysis in computer simulations to find optimum alternatives with multiple quantitative and qualitative variants. Moreover, the works of Louati et al. (2012) on people flow control based on isoarchic architecture with decision-making using multicriteria analysis and Kubler et al. (2016) regarding applications of fuzzy AHP can also be considered. From these works, it can be concluded that the AHP is one of the most used methodologies for complex decision-making considering both qualitative and quantitative variables. Nonetheless, designing control systems using the AHP to avoid and/or minimize the impact of reworks in real manufacturing processes remains an underexplored research area to date.

\section{Proposition}

The proposed hybrid control system was designed with the aim of reducing the non-quality while limiting the consumption of raw materials. To ensure this objective, Zimmermann et al. (2018) proposed a hybrid manufacturing system that combines a smart batches-based distributed control system and centralized schedule optimization. Consequently, the proposal here is to embed the batches (minimal product agent entities) with their own instance of the NN quality prediction model, to trigger it before setting up a batch on the workstation. If the risk of poor quality " $\gamma$ " is weak (under a certain threshold), then the batches are processed following the forecasted global schedule (Fig. 1). Otherwise, if the NN determines that the risk exceeds the accepted threshold for the next batch to be produced, then the system enters a collective decision with the other batches queued at the workstation (Fig. 2). The purpose of this negotiation is to determine the most suitable batch to be produced by considering the different criteria of the workstation. 


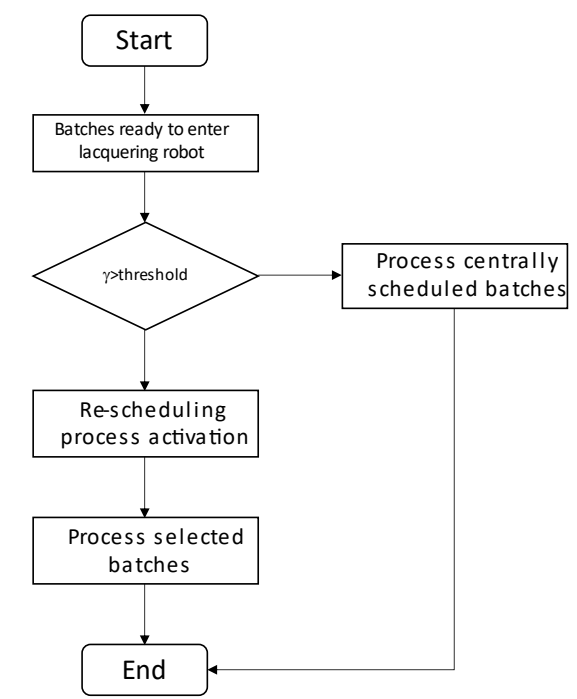

Fig. 1. Flow chart for poor-quality risk evaluation, inspired from Zimmermann et al. (2018)

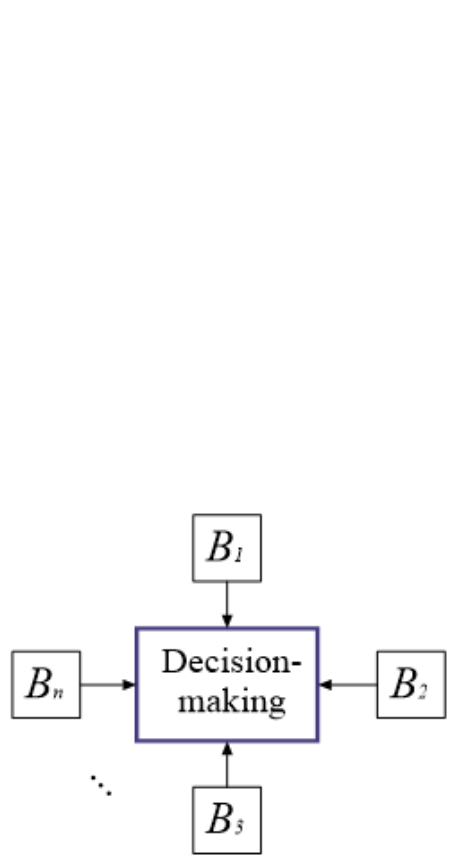

(a)

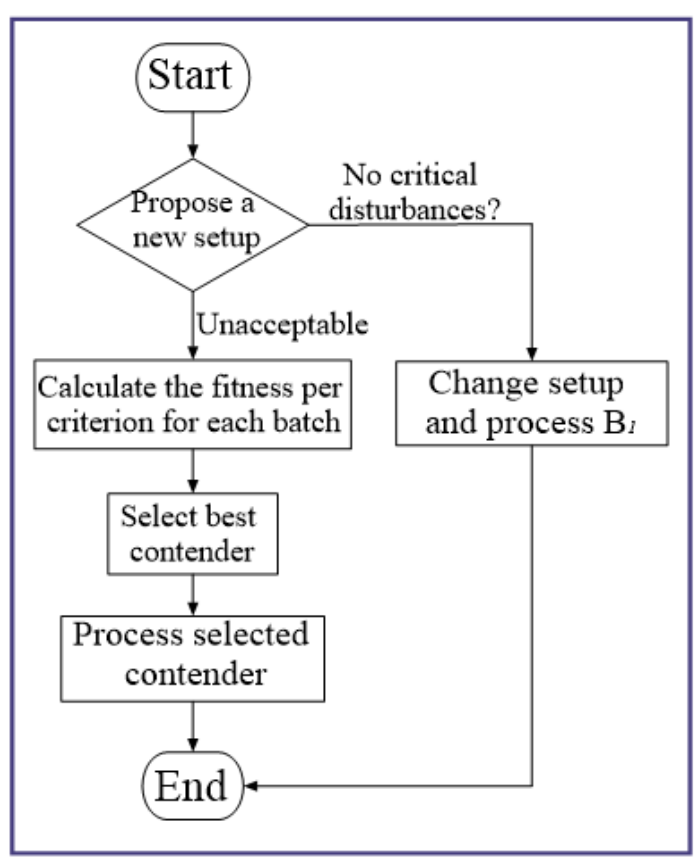

and (b)

Fig. 2. The rescheduling process: (a) set of batches, (b) flow chart for the decision-making, inspired from Zimmermann et al. (2018)

Accordingly, five criteria were selected in this proposition: 1) risk of non-quality, determined by the developed NN, 2) critical ratio (depending on customer due date), 3) balance between different product family flows, 4) adjustment times induced by the batch change, and 4) nervousness of a system (which is represented by the number of modifications made compared to original planning). The last criterion aims to limit logistical disruptions at the workstation. (i.e., the supply of lacquer, panels, or any other components necessary for machine operations). 
In the proposed AHP-based collaborative decision-making process, batches can negotiate to decide which one is going to be processed next. During the implementation of the AHP (Saaty, 1987; Shameen et al. 2020), each batch is compared with others in the queue, criterion by criterion, to build comparison matrices. Fig. 3 presents the 3-layer structure of the AHP used in this study. Level 0 represents the objective of the problem, consisting of the selection of the best batch (present in the queue) to be produced. Level 1 includes the five criteria previously described, which influence the selection in Level 0. Level 2 compares the different alternatives (five batches) of the problem, as it occurs: the batches present in the queue. However, the number of alternatives/batches to be tested can have an impact on the overall performance of the process. This problem will be studied in Section 4 (myopia). Four steps in the process must be followed. First, the criteria and alternatives matrices must be created. Then, the criteria weight vector should be computed, followed by the calculation of the alternatives weight vector. Finally, an inconsistency problem must be considered. These steps are described in the following sections.

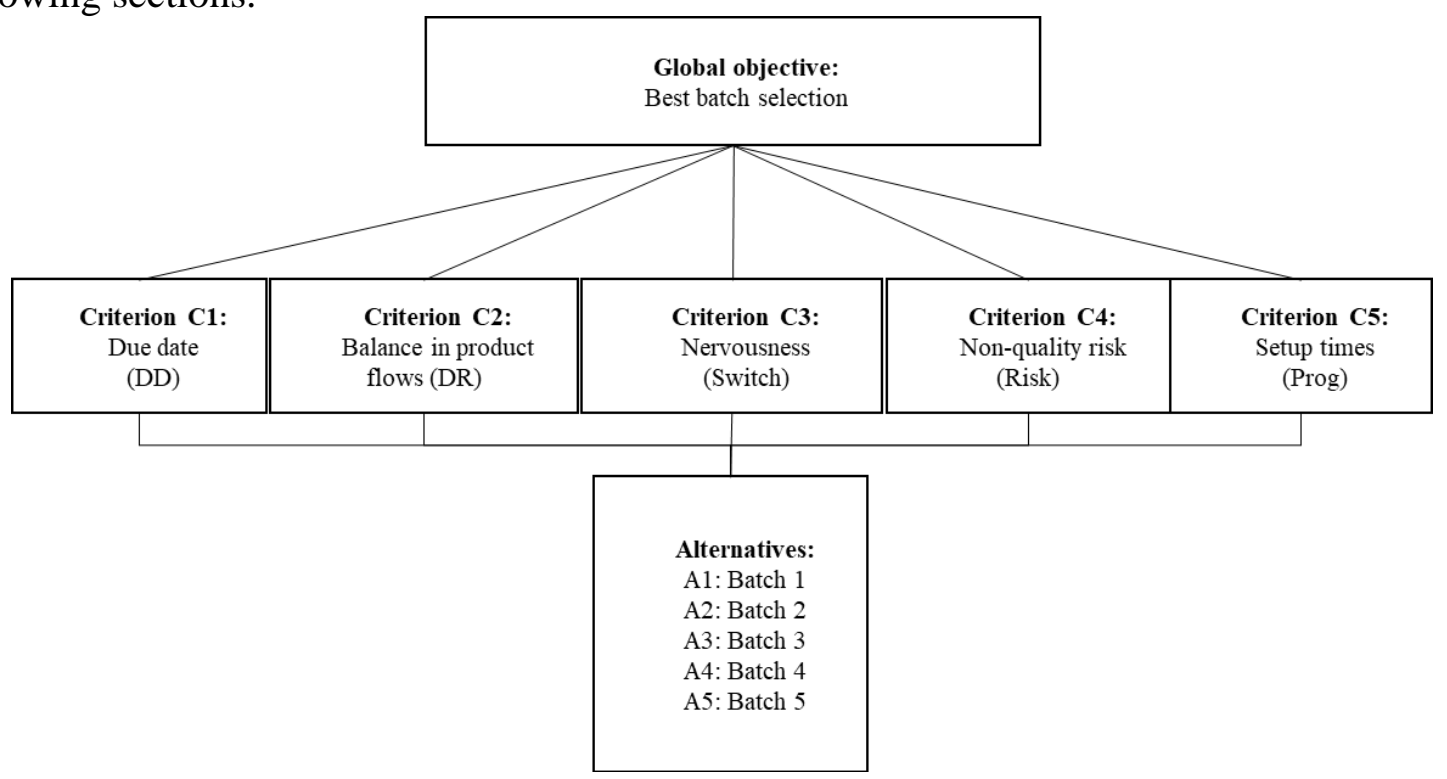

Fig. 3. The AHP model structure of the studied case

Step 1: Relative importance of the criteria and their indicators

Table 1 lists the comparison scales used in the AHP process. This comparison between two batches $i$ and $j$ is done level by level, starting with the criteria level, then with the alternatives level. We describe the transposition of this comparison scale to the five criteria mentioned below.

Table 1. Values of the decision criteria

\begin{tabular}{|l|l}
\hline Numerical value & Interpretation \\
\hline
\end{tabular}




\begin{tabular}{|l|l|}
\hline 1 & $i$ and $j$ have equal importance \\
\hline 3 & $i$ has a moderate importance compared to that of $j$ \\
\hline 5 & $i$ has a strong importance compared to that of $j$ \\
\hline 7 & $i$ has a very strong importance compared to that of $j$ \\
\hline 9 & $i$ has an extreme importance compared to that of $j$ \\
\hline
\end{tabular}

Due-date criterion:

In the case of companies delivering weekly customers, switching two batches with the same due date has no significant impact on production schedule and performance. Considering that, let $\alpha$ be the threshold set here to $4 \mathrm{~h}$ (for a semi-8-h work shift). Let $D D(k)$ and $D D(l)$ be the due dates of batches $k$ and $l$.

Table 2. Values of the alternatives regarding criterion Due date

\begin{tabular}{|c|c|}
\hline Value of $b_{k l}{ }^{(i)}$ & Interpretation \\
\hline 1 & $D D(k) \in] D D(l)-\alpha ; D D(l)+\alpha[$ \\
\hline 3 & $D D(k) \in] D D(l)-2 \alpha ; D D(l)-\alpha]$ \\
\hline 5 & $D D(k) \in] D D(l)-3 \alpha ; D D(l)-2 \alpha]$ \\
\hline 7 & $D D(k) \in] D D(l)-4 \alpha ; D D(l)-3 \alpha]$ \\
\hline 9 & $D D(k) \in]-\infty ; 0, D D(l)-4 \alpha]$ \\
\hline
\end{tabular}

Balance of product flows:

For companies with several product flows, furnisher-workstations must provide work alternatively to different customer workstations. As avoiding starvation is one of the schedule objectives, any schedule change might have an important impact on production flows, if done carelessly. In the studied case, only two production flows were considered: shiny and matt. Hence, the following formula was established:

$$
\operatorname{distance}(k)=\frac{l o a d \_s h i n y \pm l o a d \_k}{l o a d \_s h i n y+l o a d \_m a t t+\left|l o a d \_k\right|}
$$

where load_shiny and load_matt represent the shiny and matt workloads at the computation time. Then, distance $(k)$ is the ratio of the shiny workload plus or minus (plus if batch $k$ is shiny, minus if matt) the impact of batch $k$ on the total load, and $\beta$ is an objective value set at $50 \%(0.5)$. We then express the deviation from the target as follows:

$$
\operatorname{gap}(k)=|\operatorname{distance}(k)-\beta|
$$

Then, we can estimate which batch, $k$ or $l$, generates the most important impact on the global workload by calculating

$$
D R=\frac{\operatorname{gap}(k)+\varepsilon}{\operatorname{gap}(l)+\varepsilon}
$$

with $\varepsilon$ being a small value. Let also $\mu$ be the threshold set to $10 \%$.

Table 3. Values of the alternatives regarding criterion Balance of product flows

\begin{tabular}{|c|c|}
\hline Value of $b_{k l}{ }^{(i)}$ & Interpretation \\
\hline 1 & $D R \in] 1-\mu ; 1+\mu[$ \\
\hline 3 & $D R \in] 1-2 \mu ; 1-\mu]$ \\
\hline 5 & $D R \in] 1-3 \mu ; 1-2 \mu]$ \\
\hline 7 & $D R \in] 1-4 \mu ; 1-3 \mu]$ \\
\hline
\end{tabular}


$9 \quad D R \in] 0 ; 1-4 \mu]$

Nervousness:

Let $S$ witch $(k)$ be the number of position switching made with respect to the original schedule. Each time two batches are swapped, a switching counter is incremented by $1: \operatorname{Sitch}(k)+=$ 1 , with $\operatorname{Switch}(k) \in[0 ; n]$ and $n$ equal to the number of batches (5). Let $\delta$ be a threshold value set to 1 .

Table 4. Values of the alternatives regarding criterion Nervousness

\begin{tabular}{|c|c|}
\hline Value of $b_{k l}{ }^{(i)}$ & Interpretation \\
\hline 1 & Switch $(k)=\operatorname{Switch}(l)$ \\
\hline 3 & Switch $(k) \in] \operatorname{Switch}(l)-\delta ; \operatorname{Switch}(l)]$ \\
\hline 5 & Switch $(k) \in] \operatorname{Switch}(l)-2 \delta ; \operatorname{Switch}(l)-\delta]$ \\
\hline 7 & Switch $(k) \in] \operatorname{Switch}(l)-3 \delta ; \operatorname{Switch}(l)-2 \delta]$ \\
\hline 9 & Switch $(k) \in] 0 ; \operatorname{Switch}(l)-3 \delta]$ \\
\hline
\end{tabular}

Non-quality risk:

Let Risk $(k)$ be the value computed by the NN, and $\varphi$ be a threshold set up to $10 \%$. Risk represents a computation made with an NN using external parameters (such as atmospheric pressure and humidity) and internal parameters (such as color or thickness of the batch) to determine the probability of a batch having a quality defect during processing (Noyel et al., 2016).

Table 5. Values of the alternatives with respect to criterion Non-quality risk

\begin{tabular}{|c|c|}
\hline Value of $b_{k l}{ }^{(i)}$ & Interpretation \\
\hline 1 & $\operatorname{Risk}(k)=\operatorname{Risk}(l)$ \\
\hline 3 & $\operatorname{Risk}(k) \in] \operatorname{Risk}(l)-\varphi ; \operatorname{Risk}(l)]$ \\
\hline 5 & $\operatorname{Risk}(k) \in] \operatorname{Risk}(l)-2 \varphi ; \operatorname{Risk}(l)-\varphi]$ \\
\hline 7 & $\operatorname{Risk}(k) \in] \operatorname{Risk}(l)-3 \varphi ; \operatorname{Risk}(l)-2 \varphi]$ \\
\hline 9 & $\operatorname{Risk}(k) \in] 0 ; \operatorname{Risk}(l)-3 \varphi]$ \\
\hline
\end{tabular}

Setup time:

In this study, the workstation was considered as disposing of two types of setups: one for shiny products and one for matt ones. Any batch change entails a setup time. However, we must differentiate same-type batch changes $\left(b_{k l}{ }^{(i)}=1\right)$ and different-type batch changes $\left(b_{k l}{ }^{(i)}=\right.$ 5). In the first case, we can consider the setup time to be very low compared to the setup time in the second case. In this way, prog $(k)$ is the setup of batch $k$ and last_prog is the setup of the last batch that has been processed on the workstation. 
Table 6. Values of the alternatives regarding criterion Setup time

\begin{tabular}{|c|c|}
\hline Value of $b_{k l}{ }^{(i)}$ & Interpretation \\
\hline 1 & $\operatorname{prog}(k)=\operatorname{prog}(l)$ \\
\hline 5 & $\operatorname{prog}(k) \neq \operatorname{prog}(l)$ and $\operatorname{prog}(k)=\operatorname{last} \_p r o g$ \\
\hline
\end{tabular}

Step 2: Computing the vector of weights of criteria:

Let $A$ be the pairwise comparison matrix of criteria. $A$ satisfies the following constraint:

$$
a_{i j} . a_{j i}=1 \text { and } a_{i i}=1
$$

The values of $a_{i j}$ are chosen among the possibilities given in Table 1, according to the interpretation of experts in the work. In this study, all the criteria were considered equivalent to each other, and therefore all $a_{i j}$ values were set to 1 . The study led in the company so far did not allow sufficient work expertise to determine which criterion should be prioritized. However, the model could easily be improved as soon as a work expert gives his or her interpretation.

Once the matrix is built, it is possible to derive from $A$ the normalized pairwise comparison matrix $A_{\text {norm }}$ by making equal to 1 the sum of the entries on each column. Finally, the criteria weight vector $w$ (that is an m-dimensional column vector) is built by averaging the entries in each row of $A_{\text {norm }}$.

\section{Step 3: Computing the vector of weights of alternatives:}

The same work must now be done to model the links between the different alternatives and each of the criteria. In addition, for each criterion, an $n \times n$ pairwise comparison matrix $B^{(i)}$ should be established and must have the same properties as A:

$$
b_{k l}^{(i)} \cdot b^{(i)}{ }_{l k}=1 \text { and } b_{k k}^{(i)}=1
$$

The AHP applies to each matrix $B^{(i)}$ the same procedure as for the pairwise comparison matrix $A$, that is, it divides each entry by the sum of the entries in the same column and then averages the entries in each row, thus obtaining the score vectors $s^{(j)}, j=1, \ldots, m$. The vector contains the scores of the evaluated options with respect to the $j^{\text {th }}$ criterion. Once the weight vector $w$ and score matrix $S$ are computed, the AHP obtains a vector $v$ of global scores by multiplying $S$ and $w$.

\section{Step 4: Inconsistency problem:}

As explained previously, the $a_{i j}$ values of matrix $A$ were set to 1 . In this special case, the consistency problem of matrix $A$ is irrelevant. However, the matrices $B^{(i)}$ comparing the alternatives/batches will be different depending on the characteristics of the evaluated batches. Therefore, consistency must be evaluated on the score matrix $S$ for each run of the process. To do so, the $C I$ is calculated as follows:

$$
C I=\frac{x-n}{n-1}
$$

where $n$ is the number of comparisons and $x$ is the average of the values of the vector whose $i^{\text {th }}$ element is the ratio of the $i^{\text {th }}$ value of $B^{(i)} \cdot S$ to the corresponding value of $S$ is necessary. Thus, the consistency ratio is

$$
C R=\frac{C I}{R I}
$$


where $R I$ is a random evaluation value (Saaty, 1987). If the $C R$ is below 0.1 , the inconsistency is acceptable, and the best pretender is evaluated and selected. Otherwise, the original batch will be kept according to the optimization made by the global schedule. In fact, if the $C R$ is above 0.1 , the risk of inconsistency is beyond the acceptable threshold, generating small confidence in the choice of the new batch to process. In this case, it is preferred to keep confidence in the original schedule rather than risk a hazardous choice. Normally, if the $C R$ is above 0.1 , an interaction with the decision maker is required to revise the judgment. Until now, this assumption remains a proof of concept, as the method is not very elaborated. However, the objective is to have a fully automated process without the need for human contribution during execution. However, it seems possible to automatize the process of matrix correction as soon as $C R>0.1$.

The pseudocode of the AHP process is derived from that proposed by Saaty (1987). The main difference with the standard AHP lies in the determination of the matrices $B^{(i)}$ to compare the alternatives; each run of the process requires the elaboration of new pairwise comparison matrices, as each batch has its own surface, color, due date, and setup. In addition, for each computation, the environment is different: workflows and non-quality risks are continuously evolving, depending on external or internal parameters.

Algorithm of the AHP:

\section{Inputs:}

$n$ : number of criteria and number of alternatives

$A: n \times n$ criteria matrix

Outputs:

Return a number between 1 and $n$ corresponding to the selected batch

$$
\begin{aligned}
& S=n \times n / / \text { score matrix } \\
& B^{(i)}=n \times n / / \text { pairwise comparison matrix for criterion } \mathrm{i} . \\
& w=1 \times n / / \text { weight vector } \\
& \bar{A}=\left(\begin{array}{ccc}
\overline{a_{11}} & \ldots & \overline{a_{n n}} \\
\vdots & \ddots & \vdots \\
\overline{a_{n 1}} & \ldots & \overline{a_{n n}}
\end{array}\right) \text { with } \overline{a_{i j}}=\frac{a_{i j}}{\sum_{l=1}^{n} a_{l j}} \\
& w_{j}=\frac{\sum_{l=1}^{n} \overline{a_{j l}}}{n} \\
& \text { For } \left.i:=1 \text { to } n \quad \begin{array}{ccc}
1 & \ldots & 1 \\
\vdots & \ddots & \vdots \\
1 & \cdots & 1
\end{array}\right) \\
& B^{(i)}=\begin{array}{l}
1 \text { to } n \\
\text { For } k:=1 \text { to } n
\end{array} \\
& \text { For } j:=
\end{aligned}
$$




$$
\begin{aligned}
b_{j k}^{(i)} & =\text { compare_AHP( } i, j, k) \quad / / \text { is elaborated dynamically. } \\
b_{k j}^{(i)} & =\frac{1}{b^{(i)}{ }_{j k}}
\end{aligned}
$$

\section{End for}

End for

$$
\begin{gathered}
{\overline{b^{(i)}}}_{j k}=\frac{b^{(i)}{ }_{j k}}{\sum_{l=1}^{n} b^{(i)} l k} \\
s_{j k}=\frac{\sum_{l=1}^{n} \overline{b_{k l}}}{n}
\end{gathered}
$$

\section{End for}

$$
\begin{gathered}
\text { If } \frac{C I}{R I}<0.1 \text { then } / /(\text { with } I R=1.12 \text { for } n=5) \\
v=S w
\end{gathered}
$$

Else

$$
\text { Return } \max \left(v_{i}\right)
$$

End

\section{End if}

Return $1 / /$ the schedule is not altered and the first batch is chosen

\section{Case study: Industrial applicability}

\subsection{Description of the industrial context}

As highlighted in Section 3, the proposition deals with the specific case of a lacquering robot in the Acta Mobilier company. This company suffers from a high rework rate, above 30\%, owing to technological limitations and the high degree of product quality expectations from customers. The lacquering robot feeds two separate product flows:

- "Matt" products, directly delivered to the shipment station.

- "Shiny" (or "high gloss" in Fig. 4) products, which are processed by the polishing machine before joining the other product type in the shipping station (Fig. 4).

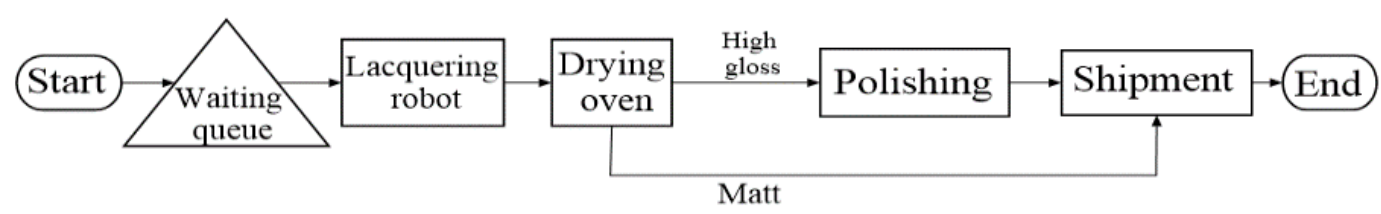

Fig. 4.: Flow chart of different product flows for the lacquering robot in Acta Mobilier (Zimmermann et al., 2018)

\subsection{Validation approach}

The Intelligent Manufacturing Systems-Network of Excellence (IMS-NoE) has shown the relevance of simulation-based benchmarking in estimating the efficiency of plant-wide control and organizational issues before their practical deployment into complex manufacturing systems (Cavalieri et al., 2003; Valckenaers et al., 2006). For this reason, a Python-based simulation model was developed to represent the lacquering workshop and its queue. In the simulation, the queue was set to five batches. These batches participate in the decision-making 
process for the best pretender. This has been modeled by five entering queues, numbered from $q_{1}$ to $q_{5}$, with the capacity to process one single batch. These five queues can collaborate in the case of non-quality risk detection, as presented in Section 3. Every time a batch is completed on the workstation, a quality check is performed. If the predicted quality satisfies the acceptability range, it leaves the queue, and a new product enters. If not, it puts itself back into the queue.

The sequence for product entry in the queue is determined by the optimal predictive schedule. In the normal state, queue $q_{5}$ is supposed to be processed next. Fig. 5 illustrates this scenario: batches $B_{1}$ to $B_{5}$ are sorted according to the predictive global schedule and without poor-quality detection. $B_{1}$ is the first batch to be processed if no poor-quality risk is detected. Each time a batch enters a machine, the others move to the next queue. Then, according to the product information, the robot realizes the corresponding program, and the batch leaves the machine with the remaining time reduced by drying time $(7 \mathrm{~h})$. Note that the processing time is proportional to the global panel surface in the batch. When the batch exits the system, a random number is generated to determine whether it is defective. If defective, it returns to the input queue to be reworked. Otherwise, it exits the machine and goes to one of the two possible downstream queues: high gloss or matt (Fig. 4).

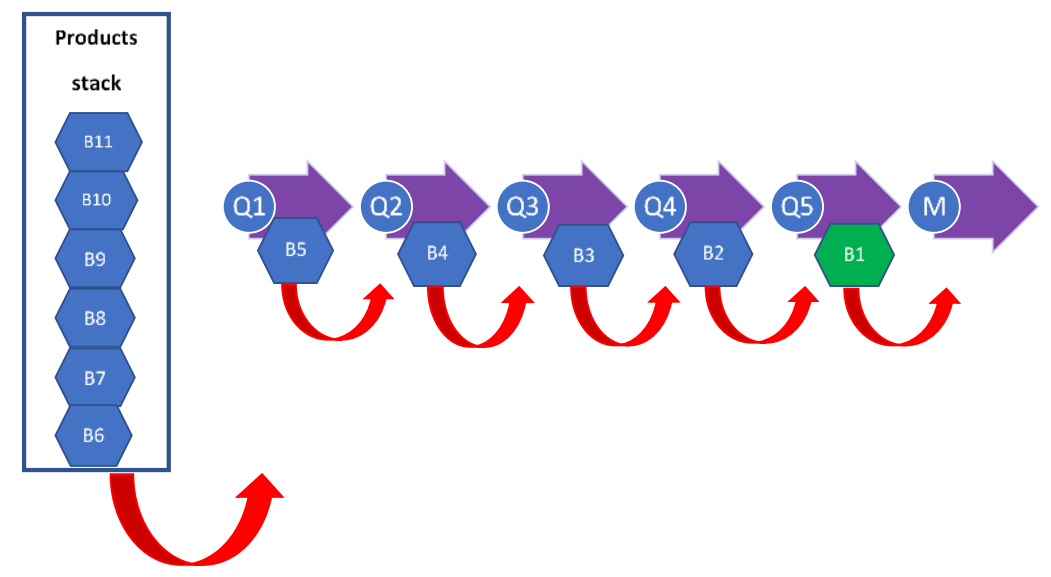

Fig. 5. Flow in a stable environment (Zimmermann et al., 2018)

Before entering the lacquering robot, batch $B_{1}$ in $q_{5}$ launches the computation of its embedded $\mathrm{NN}$ to determine its non-quality risk. If the risk is higher than the threshold of $25 \%$, as established in Section 3, the AHP-based application is recalled, addressing the procedure for selecting the best replacement. The algorithm presented in Section 3 is then launched to determine which batch best corresponds to the five optimization criteria. Accordingly, Fig. 6 shows a scenario where the NN of $B_{1}$ has evaluated a very heavy risk of potential non-quality 
for the initial batch entry order in the decisional-based AHP process and, as a result, rescheduling puts $B_{3}$ in the first position to achieve better overall quality.

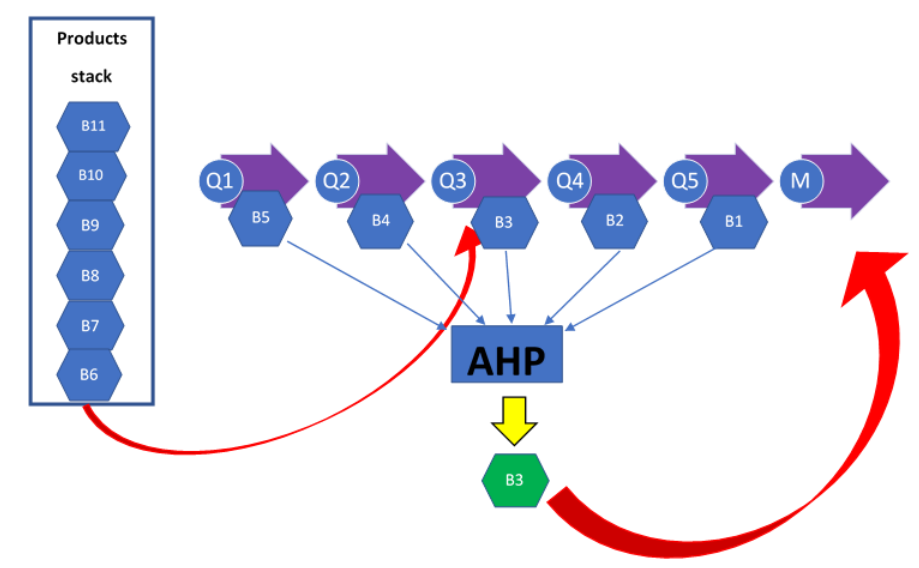

Fig. 6. Flow in case of non-quality risk detection (Zimmermann et al., 2018)

The variables of this new model are listed below, and Table 7 presents a summary of their numerical values.

- Variables related to production range:

- "Area" is the total surface of the products composing the batch, expressed in $\mathrm{m}^{2}$.

- "Program" is the number of the program the machine should use to realize its operation on a batch (for example: 2:2 means that program number 2 will lead to process the two wood panel sides with shiny paint).

- "Due date" is the batch due date, expressed in hours.

- "Number of pass" expresses the number the batch processing loops on the robot.

- Variables related to the workstation:

- The four programs (P1, P2, P3, and P4) with their own duration times are expressed in $h / m^{2}$.

- The two possible setup times, expressed in hours:

- $\mathrm{S} 0$ if the last batch and next batch program are in the subset $\{\mathrm{P} 1, \mathrm{P} 2\}$ or in the subset $\{\mathrm{P} 3, \mathrm{P} 4\}$.

- S1 elsewhere (corresponding to a finishing change).

○ "Defect happening" counts the number of defectives batches produced.

Table 7. Summary of variables of the new model

\section{Products}

\begin{tabular}{rll}
\hline Area & Float & {$[2.5: 40]$} \\
\cline { 2 - 3 } Program & Integer & $1: 1 \Rightarrow$ high-gloss side \\
& & $2: 2 \Rightarrow$ high-gloss sides \\
\hline
\end{tabular}




\begin{tabular}{|c|c|c|}
\hline Robot & & \\
\hline Program PI & & 0.28 \\
\hline Program $P 2$ & $\mathrm{~h} / \mathrm{m}^{2}$ & 0.04 \\
\hline Program P3 & & 0.03 \\
\hline Program P4 & & 0.02 \\
\hline $\begin{array}{l}\text { Setup without } \\
\text { finishing changes } \\
\text { SO }\end{array}$ & $\mathrm{h}$ & 0.03 \\
\hline $\begin{array}{r}\text { Setup with } \\
\text { finishing changes } \\
\text { S1 }\end{array}$ & $\mathrm{h}$ & 0.05 \\
\hline
\end{tabular}

\section{Model}

Defect rate Float $\quad[0: 100]$

\subsection{Experimental protocol and discussions}

To validate the proposed method, simulation experiments were conducted under several distinct conditions, with and without the use of the proposed AHP-based approach and considering a constant number of decision participants. For each case, a set of one thousand simulations were performed. The means and standard deviations $(s t d)$ for the FIFO and AHP cases are presented in Table 8. Hence, to draw the benefits of the AHP method over the others, a global cost function (expressed in euros) described as the weighted sum of the following three KPIs (also expressed in euros) is compared:

- Lateness represents the number of overdue hours multiplied by the penalty cost. It is an approximate way to express the cost of the lateness, which is complex to represent, because a product in overdue implies a heavy impact on the company brand-building, a loss of customer confidence and, in some cases, a complementary truck departure.

- The rework cost, which is the cost implied by the fact of a re-working batch on the robot and is taken directly from the Acta Mobilier company actual data. 
- The workforce cost is calculated by multiplying the cost in man-days of two operators (the minimal number of human operators needed to pilot the robot) by a makespan.

Hence, Table 8 highlights that the AHP algorithm provides a better solution for a nonoptimized dataset. For example, the rework cost in the AHP is $25 \%$ lower than that in the FIFO scheduling approach, for a global reduction of $26 \%$.

Table 8. Comparing FIFO and AHP in an actual case

\begin{tabular}{|l|r|r|r|r|r|r|r|r|}
\hline \multirow{2}{*}{ Real case } & \multicolumn{2}{|c|}{ Lateness } & \multicolumn{2}{c|}{ Rework } & \multicolumn{2}{c|}{ Operating cost } & \multicolumn{2}{c|}{ global cost } \\
\cline { 2 - 9 } & mean & std & mean & std & mean & std & mean & std \\
\hline FIFO & 389.8 & 549.0 & 5346.5 & 4175.0 & 161.5 & 25.4 & 5897.8 & 4449.2 \\
\hline AHP & 175.8 & 350.5 & 4025.1 & 3472.7 & 149.7 & 20.5 & 4350.6 & 3619.4 \\
\hline
\end{tabular}

The first case studied is an actual example of the production of a lacquering robot without any optimization approach. The production of a team (eight working hours per day) was used in this study. Then, a simulation test with an optimal schedule having an equal balance of the gloss and matt flows is used to determine the benefit of the proposal placed in the optimal possible case. Hence, from Table 9, it can be concluded that when a data set is already scheduled to create a good balance between the two product flows to be supplied, the AHP method still offers a rework improvement of $21 \%$ for a global enhancement of $20 \%$.

Table 9. Equal distribution condition

\begin{tabular}{|l|r|r|r|r|r|r|r|r|}
\hline \multirow{2}{*}{$\begin{array}{c}\text { Equal } \\
\text { distribution }\end{array}$} & \multicolumn{2}{|c|}{ Lateness } & \multicolumn{2}{c|}{ Rework } & \multicolumn{2}{c|}{ Operating cost } & \multicolumn{2}{c|}{ global cost } \\
\cline { 2 - 9 } & mean & std & mean & std & mean & \multicolumn{1}{c|}{ std } & \multicolumn{1}{c|}{ mean } & std \\
\hline FIFO & 0.0 & 0.0 & 1791.5 & 1036.5 & 89.8 & 13.9 & 1881.3 & 1048.5 \\
\hline AHP & 0.0 & 0.0 & 1417.1 & 876.1 & 82.4 & 10.0 & 1499.5 & 884.4 \\
\hline
\end{tabular}

Then, the following two tests were conducted to determine if the solution remained effective when one of the decision criteria was defective.

a) Under an extreme load distribution (only high-gloss products or only matt products), which also impacts the minimization of the setup time (see Table 10). Although the system faces this situation, the result obtained using the AHP is still improved by $18 \%$ compared to that of the FIFO method for only high gloss and $25 \%$ for only matt.

b) As soon as all products set the same due date with an optimized schedule, the benefits brought by the AHP are almost exclusively on the rework cost and to some extent on the lateness, as indicated in Table 11. At this time, the improvement is recorded as $23 \%$.

Table 10. Extreme load distribution condition 


\begin{tabular}{|c|c|c|c|c|c|c|c|c|}
\hline \multirow{2}{*}{$\begin{array}{c}\text { Only high } \\
\text { gloss }\end{array}$} & \multicolumn{2}{|c|}{ Lateness } & \multicolumn{2}{|c|}{ Rework } & \multicolumn{2}{|c|}{ Operating cost } & \multicolumn{2}{|c|}{ global cost } \\
\hline & mean & std & mean & std & mean & std & mean & std \\
\hline FIFO & 10.6 & 61.8 & 3312.5 & 2083.5 & 117.9 & 17.8 & 3441.0 & 2124.9 \\
\hline $\mathrm{AHP}$ & 1.4 & 19.6 & 2694.4 & 1748.4 & 109.5 & 13.3 & 2805.3 & 1761.7 \\
\hline \multirow[t]{2}{*}{ Only mat } & \multicolumn{2}{|c|}{ Lateness } & \multicolumn{2}{|c|}{ Rework } & \multicolumn{2}{|c|}{ Operating cost } & \multicolumn{2}{|c|}{ global cost } \\
\hline & mean & std & mean & std & mean & std & mean & std \\
\hline FIFO & 0.0 & 0.4 & 3350.5 & 2015.4 & 101.6 & 14.5 & 3452.0 & 2027.0 \\
\hline $\mathrm{AHP}$ & 0.0 & 0.0 & 2747.6 & 1894.1 & 94.6 & 12.2 & 2842.2 & 1903.5 \\
\hline
\end{tabular}

Table 11. Same due-date condition

\begin{tabular}{|l|r|r|r|r|r|r|r|r|}
\hline \multirow{2}{*}{$\begin{array}{c}\text { Same due } \\
\text { date }\end{array}$} & \multicolumn{2}{|c|}{ Lateness } & \multicolumn{2}{c|}{ Rework } & \multicolumn{2}{c|}{ Operating cost } & \multicolumn{2}{c|}{ global cost } \\
\cline { 2 - 9 } & mean & \multicolumn{1}{c|}{ std } & mean & std & mean & \multicolumn{1}{c|}{ std } & \multicolumn{1}{c|}{ mean } & std \\
\hline FIFO & 0.4 & 7.8 & 5054.0 & 4015.8 & 161.1 & 26.1 & 5215.4 & 4037.5 \\
\hline AHP & 2.3 & 37.4 & 3855.5 & 3556.4 & 149.5 & 21.0 & 4007.2 & 3587.6 \\
\hline
\end{tabular}

\subsection{Social myopia of smart batches analysis}

In Subsection 4.3, the performance of the proposed method should be closely linked to its settings (e.g., weights of the criteria and number of actors in the entire decision-making process). However, validation was performed considering a constant number of batches as decisional participants (one product representing one batch). Now, the focus will be on estimating the impact of the number of decision process participating entities on overall quality. Therefore, to measure the impact of social myopia, the number of decisional products (i.e., batches in the queue) is set as a variable. To do so, the simulation was re-launched, running on the data set corresponding to a real production week for the lacquering robot. The weights of all the criteria were set to 1 , and only the number of products involved in the decision-making process varied.

Accordingly, simulation tests were performed considering 1, 3, 5, 7, 10, and 20 batches. The two extreme values (i.e., 1 batch and 20 batches) were used to obtain extreme values, even if they were irrelevant. Indeed, one single batch can only communicate with itself, implying that no real decision-making process can be performed. If the lacquering robot does not operate in pull flow with its upstream station, this situation rarely occurs. Likewise, it is highly unlikely for this workstation to have a queue with more than 10 products, except in the case of extreme congestion caused by machine failure. Figures 7 and 8 summarize the results obtained for the above-mentioned situations (where $m$ is the number of batches in the queue). As expected, the results are the poorest when a single batch is involved in the decision process. 


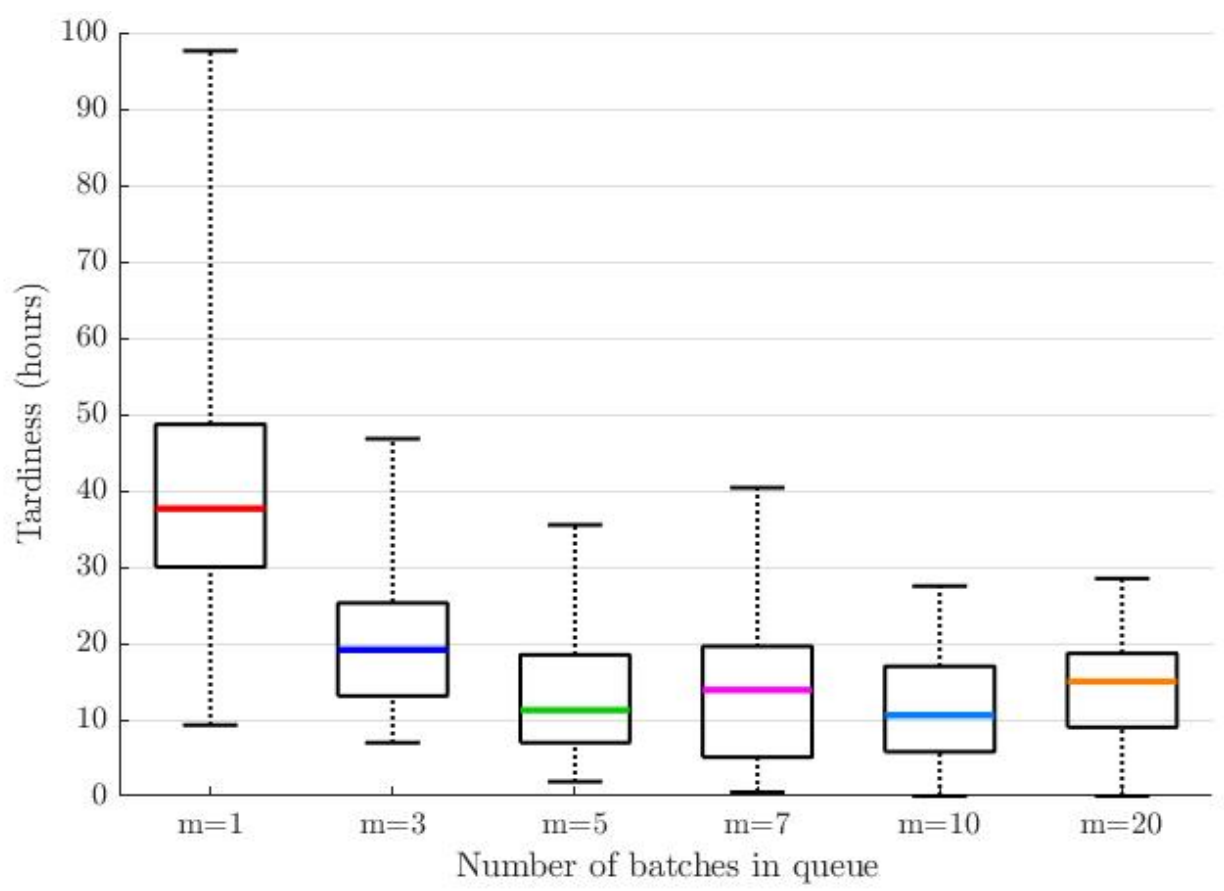

Fig. 7. Impact of social myopia on delay (tardiness) (in hours)

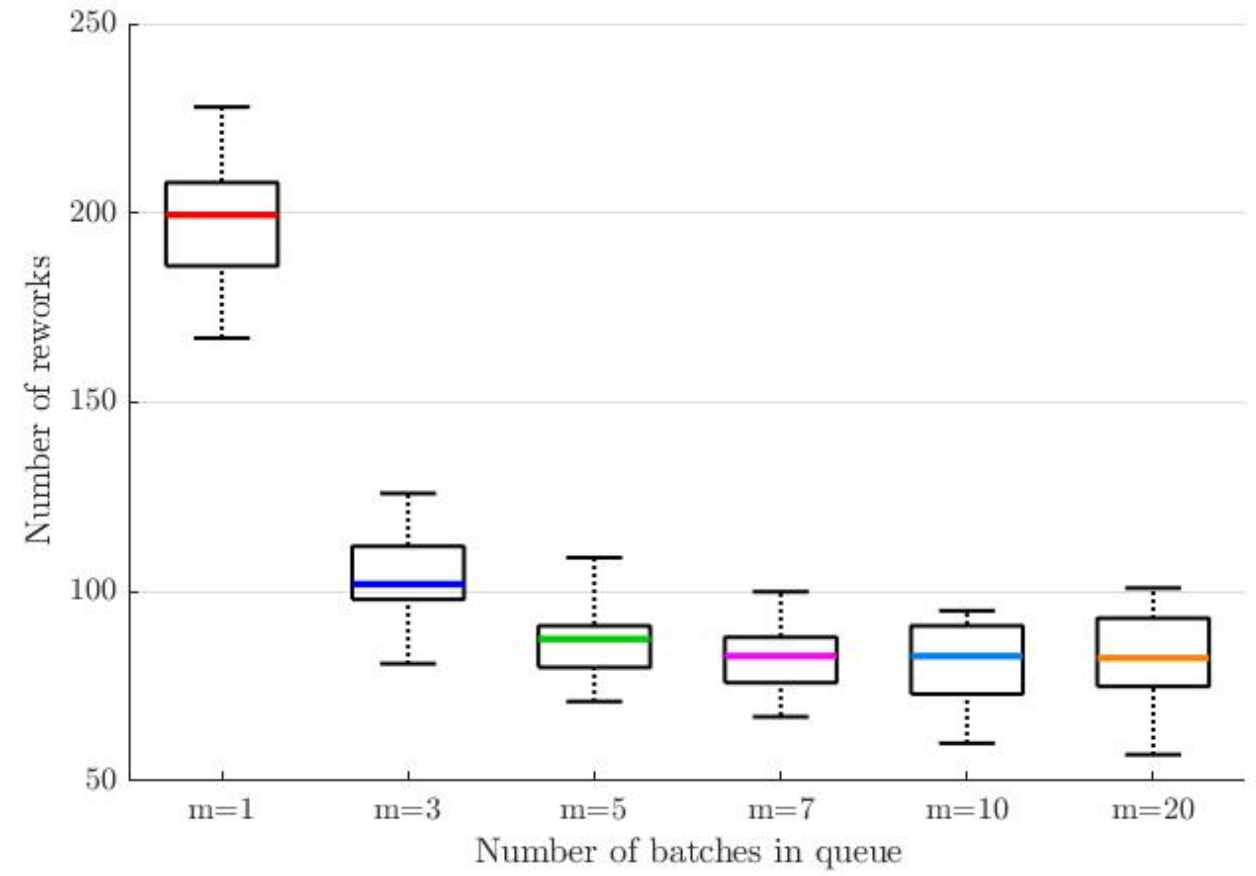

Fig. 8. Impact of social myopia on number of reworks

From Figure 9, it can be noted that for more than five batches in the queue, the average results stagnate as the computing time increases. Hence, in view of the results and having knowledge of the evolution of the usual flow at the entrance of the lacquering robot, the choice of five 
communicating batches for decision-making under standard operating conditions seems to be the most relevant.

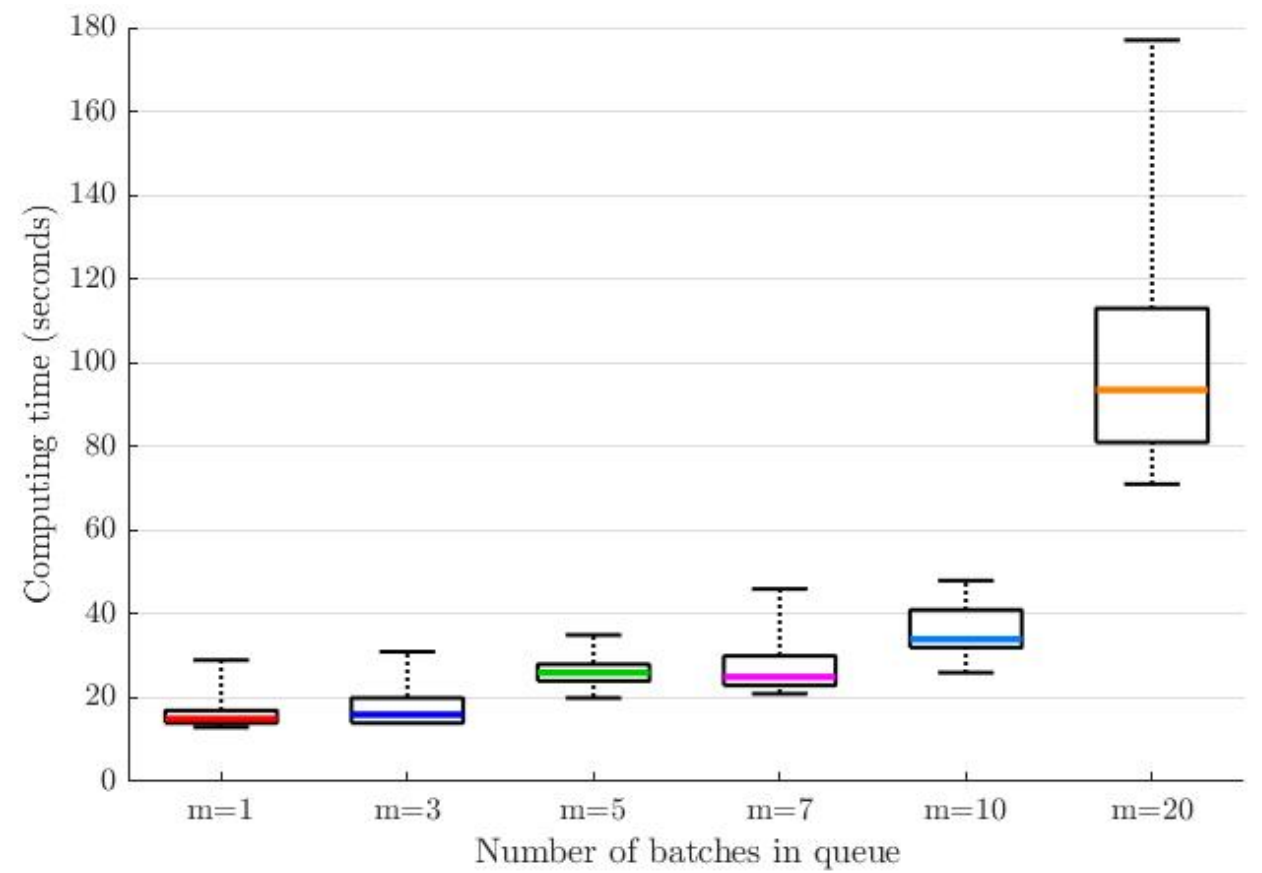

Fig. 9. Impact of social myopia on computing time (in seconds)

To better analyze the results, a one-dimensional variance analysis (ANOVA) was used to measure the effect of the change in modality of 'myopia' on the number of reworks, cumulative delay, and time calculation. To apply ANOVA, some recommendations must be verified.

- A single variability factor (myopia) with six modalities ( $m=1, m=3, m=5, m=7, m$ $=10$, and $m=20$ )

- Random and independent draws

- Observations supposed without errors.

The ANOVA test involved comparing two different modalities of the factor. It generates a $p$-value that must be compared to the value of the risk $\alpha$ that we have to choose (here taken at $5 \%$ ). The two hypotheses are as follows:

- H0: The two considered modalities of the factor studied have the same impact on the variable.

- H1: The two considered modalities of the factor studied have different impact. 
Accordingly, the result of the test is obtained by comparing the $p$-value with the value of the chosen risk $\alpha$. That is, if the $p$-value is greater than risk $\alpha(p>0.05)$, then $\mathrm{H} 0$ is accepted with a risk, if the $p$-value is less than risk $\alpha(p<0.05)$, then $\mathrm{H} 0$ is rejected.

From Table 11, we can observe that $\mathrm{H} 0$ is systematically rejected when modalities 1 and 3 are at stake. Consequently, choosing one of these two modalities has a significant impact on the result. When comparing modalities $5,7,10$, and $20, \mathrm{H} 0$ is systematically accepted (except for case 5-10, which still gives a value close to the threshold). Therefore, choosing between modalities 5, 7, 10, and 20 does not have any impact on the number of reworks. Thus, setting modality at 5 seems to be the best compromise regarding the number of reworks.

In addition, relative to the impact of myopia on delay in Table 12, we can notice that $\mathrm{H} 0$ is systematically rejected when modalities 1 and 3 are at stake. Hence, choosing one of these two modalities has a significant impact on the result. When comparing modalities 5, 7, 10, and 20 sets, H0 is systematically accepted and choosing between modalities 5, 7, 10, and 20 has no different impact on the delay. Thus, setting this modality at 5 also seems to be the best compromise in terms of delay.

Finally, corresponding to the impact of myopia on the time calculation in Table 13, it can be noticed that $\mathrm{H} 0$ is rejected for all combinations except for the two modality pairs, 1-3 and 5-7.

Table 11. ANOVA test results for reworks

\begin{tabular}{|c|c|c|c|c|c|c|c|}
\hline & Myopia & 1 & 3 & 5 & 7 & 10 & 20 \\
\hline \multirow{5}{*}{ 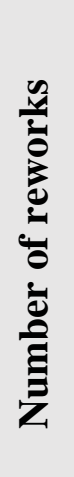 } & 1 & - & 0 & 0 & 0 & 0 & 0 \\
\hline & 3 & & - & 0 & 0 & 0 & 0 \\
\hline & 5 & & & - & 0.1691 & 0.0457 & 0.1755 \\
\hline & 7 & & & & - & 0.4085 & 0.8351 \\
\hline & 10 & & & & & - & 0.6017 \\
\hline
\end{tabular}

Table 12. ANOVA test results for delay/tardiness

\begin{tabular}{|c|c|c|c|c|c|c|c|}
\hline & Myopia & 1 & 3 & 5 & 7 & 10 & 20 \\
\hline 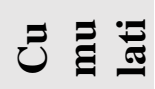 & 1 & - & 0 & 0 & 0 & 0 & 0 \\
\hline
\end{tabular}




\begin{tabular}{cccccc}
\hline $\mathbf{3}$ & - & 0.0148 & 0.0079 & 0.0001 & 0.0100 \\
\hline $\mathbf{5}$ & & - & 0.8122 & 0.1562 & 0.9441 \\
\hline $\mathbf{7}$ & & - & 0.2431 & 0.7420 \\
\hline $\mathbf{1 0}$ & & & - & 0.0996 \\
\hline
\end{tabular}

Table 13. ANOVA test results for time calculation

\begin{tabular}{|c|c|c|c|c|c|c|c|}
\hline & Myopia & 1 & 3 & 5 & 7 & 10 & 20 \\
\hline \multirow{5}{*}{ 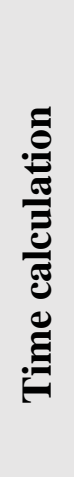 } & 1 & - & 0.1296 & 0 & 0 & 0 & 0 \\
\hline & 3 & & - & 0 & 0 & 0 & 0 \\
\hline & 5 & & & - & 0.4788 & 0 & 0 \\
\hline & 7 & & & & - & 0 & 0 \\
\hline & 10 & & & & & - & 0 \\
\hline
\end{tabular}

\section{Conclusions and outlooks}

In this work, we have demonstrated the interest of using a hybrid manufacturing control system based on a predictive schedule and the AHP multicriteria decision method as a decision support 
tool in the context of a company suffering from a high rework rate. The consideration of the number of batches that participate in the decision (social myopia) is proven to be important and has a considerable impact on the quality and calculation time. The AHP method is used dynamically when disturbances occur or when the smart batches using the embedded NN algorithm estimate a high risk of non-quality. The analysis of myopia allows us to consider an evolutionary number of batches participating in the decisions, depending on the quality of the results. The real number of batches in the queue of the workstation should be considered.

Even if the simulation results show interesting improvement of different KPIs, there are still many issues and complementary scenarios to explore. Future work might, for example, use a larger dataset from the company to evaluate the behavior of the shop floor over a longer period. Furthermore, harvesting more data from the simulations could lead to a self-adapting pairwise comparison matrix constructed by an active learning approach. In addition to costs, the profiles of the high gloss and matt loads in the case study should be analyzed. Further, complements should be provided to the model to make it even more realistic. For instance, the consumption speed of the two output queues should be added to the model. To go further, a complementary study of the weight to give to each criterion must be led with the company experts after the application practical feedback. The fact that other workstations also feed them and that different types of reworks with different process times may occur (from simple correction to total reproduction) should also be considered.

\section{Acknowledgements}

Authors would like to thank the company, for the case study and provided dataset. They also thank the reviewers for their contributions, which helped to improve significantly this paper.

\section{References}

Azadeh A., Ghaderi S.F., Izadbakhsh H., 2008. Integration of DEA and AHP with computer simulation for railway system improvement and optimization, Appl. Math. Comput., 195(2), 775-785.

Barbosa J., Leitão P., Adam E., Trentesaux D., 2015. Dynamic self-organization in holonic multi-agent manufacturing systems: The ADACOR evolution. Computers in Industry, 66, 99-111.

Bian, T., Hu, J., \& Deng, Y. (2017). Identifying influential nodes in complex networks based on AHP. Physica A: Statistical Mechanics and its Applications, 479, 422-436.

Büyüközkan G., Alpay Havle C., Feyzioğlu O., 2021 Digital competency evaluation of lowcost airlines using an integrated IVIF AHP and IVIF VIKOR methodology. Journal of Air Transport Management, Volume 91, 101998.

Cardin, O., Trentesaux, D., Thomas, A. et al., 2017 Coupling predictive scheduling and reactive control in manufacturing hybrid control architectures: state of the art and future challenges. J Intell Manuf 28, 1503-1517

Cavalieri S., Macchi M., Valckenaers P., 2003. Benchmarking the performance of manufacturing control systems: Design principles for a web-based simulated testbed. Journal of Intelligent Manufacturing, 14(1), 43-58. 
Chan F., Chung S., Wadhwa S., 2005. A hybrid genetic algorithm for production and distribution, Omega, 33(4), 345-355.

Cheng E.W., Li H., Ho D.C., 2002. Analytic hierarchy process (AHP). Measuring Business Excellence.

Hanssmann F., Hess S.W., 1960. A Linear Programming Approach to Production and Employment Scheduling," Manag. Technol., vol. 1, no. 1, pp. 46-51.

Held M., Karp R.M., 1962. A Dynamic Programming Approach to Sequencing Problems, J. Soc. Ind. Appl. Math., 10(1), 196-210.

Indriago C., Cardin O., Rakoto N., Castagna P., \& Chacòn E. 2016. H2CM: A holonic architecture for flexible hybrid control systems. Computers in Industry, 77, 15-28.

Jamal A.M.M., Sarker B.R., Mondal S., 2004. Optimal manufacturing batch size with rework process at a single-stage production system. Computers \& industrial engineering, 47(1), 77-89.

Jimenez J.F., Bekrar A., Zambrano-Rey G., Trentesaux D., Leitão P., 2017. Pollux: a dynamic hybrid control architecture for flexible job shop systems. International Journal of Production Research, 55(15), 4229-4247.

Liu F.H.F., Hai H.L., 2005. The voting analytic hierarchy process method for selecting supplier. International journal of production economics, 97(3), 308-317.

Louati, T., Ounnar, F., Pujo, P., \& Pistoresi, C., 2012. Multicriteria decision making service for an intelligent control of individuals flow in restricted area. IFAC Proceedings Volumes, 45(6), 685-690.

Mezgebe T.T., Demesure G., El Haouzi H.B., Pannequin R., Thomas A., 2019. CoMM: a consensus algorithm for multi-agent-based manufacturing system to deal with perturbation. International Journal of Advanced Manufacturing Technology, 105(9), 3911-3926.

Momoh J.A., Zhu J., 2003. Optimal generation scheduling based on AHP/ANP, IEEE Trans. Syst. Man Cybern. Part B Cybern., 33(3), 531-535.

Moslem, S., Ghorbanzadeh, O., Blaschke, T., \& Duleba, S., 2019. Analysing stakeholder consensus for a sustainable transport development decision by the fuzzy AHP and interval AHP. Sustainability, 11(12), 3271.

Noyel M., Thomas P., Thomas A., Charpentier P., 2016. Reconfiguration process for neuronal classification models: Application to a quality monitoring problem, Computers in Industry, 83, 78-91.

Pach C., Berger T., Bonte T., Trentesaux D., 2014. ORCA-FMS: a dynamic architecture for the optimized and reactive control of flexible manufacturing scheduling. Computers in Industry, 65(4), 706-720.

Parsons S., Wooldridge M., 2002. Game theory and decision theory in multi-agent systems. Autonomous Agents and Multi-Agent Systems, 5(3), 243-254.

Pitt J., Kamara L., Sergot M., Artikis E. 2006. Voting in Multi-Agent Systems, The Computer Journal, 49, 156-170. 
Quintanilla F.G., Cardin O., L'anton A., Castagna P., 2016. A modeling framework for manufacturing services in service-oriented holonic manufacturing systems. Engineering Applications of Artificial Intelligence, 55, 26-36.

Rey, G. Z., Bonte, T., Prabhu, V., \& Trentesaux, D. (2014). Reducing myopic behavior in FMS control: A semi- heterarchical simulation-optimization approach. Simulation Modelling Practice and Theory, 46, 53-75.

Saaty R.W., 1987. The analytic hierarchy process-What it is and how it is used. Mathl Modelling, Vol 9. No. 3-5, pp 161-176,1987.

Saaty T.L., 1980, The analytic hierarchy process, McGraw Hill

Saaty T.L., 2008. Decision making with the analytic hierarchy process. International journal of services sciences, 1(1), 83-98.

Saaty, T. L., \& Tavana, M., 2021. The Encyclicon-Volume 4: A Dictionary of Complex Decisions Using the Analytic Network Process. RWS Publications.

Schmickl T., Thenius R., Moeslinger C., Radspieler G., Kernbach S., Szymanski M., Crailsheim K., 2009. Get in touch: cooperative decision making based on robot-to-robot collisions, Auton. Agents Multi-Agent Syst., 18(1), 133-155.

SHAMEEM, Mohammad, KHAN, Arif Ali, HASAN, Md Gulzarul, et al. Analytic hierarchy process based prioritisation and taxonomy of success factors for scaling agile methods in global software development. IET Software, 2020, vol. 14, no 4, p. 389-401.

Teodorovic D., 2003. Transport modeling by multi-agent systems: a swarm intelligence approach. Transportation planning and Technology, 26(4), 289-312.

Thomas P., El Haouzi H., Suhner, M.-C. Thomas A., Zimmermann E., Noyel M., 2018. Using a Classifier Ensemble for Proactive Quality Monitoring and Control: the impact of the choice of classifier types, selection criterion, and fusion process. Computers in Industry. 30 .

Vaidya O.S., Kumar S., 2006. Analytic hierarchy process: An overview of applications. European Journal of operational research, 169(1), 1-29.

Valckenaers P., Cavalieri S., Sint Germain B., Verstraete P., 2006. A benchmarking service for the manufacturing control research community. Journal of Intelligent Manufacturing, 17(6), 667-679.

Valckenaers P., 2018. ARTI reference architecture-PROSA revisited. International Workshop on Service Orientation in Holonic and Multi-Agent Manufacturing, Springer, Cham, 1-19.

Wee H.M., Widyadana G.A., 2013. A production model for deteriorating items with stochastic preventive maintenance time and rework process with FIFO rule. Omega, 41(6), 941-954.

Xiang W., Lee H.P., 2008. Ant colony intelligence in multi-agent dynamic manufacturing scheduling, Eng. Appl. Artif. Intell., 21(1), 73-85.

Zimmermann E., El Haouzi H., Thomas P., Thomas A., Noyel M., 2017. A hybrid manufacturing control based on smart lots in a disrupted industrial context, in $20^{\text {th }}$ IFAC World Congress, IFAC 2017.

Zimmermann E., El Haouzi H., Thomas P., Pannequin R., Noyel M., 2018. Using analytic hierarchical process for scheduling problem based on smart lots and their quality prediction capability. $8^{\text {th }}$ Workshop on Service Orientation in Holonic and Multi Agent Manufacturing Control SOHOMA'18, Bergame 